\title{
A Research of the Constructing of Cultural Field of Danmu Video Website Bilibili in China
}

\author{
Tian Yang \\ School of Journalism and Communication, Renmin University, Beijing, China
}

\section{Email address:}

498378486@qq.com

\section{To cite this article:}

Tian Yang. A Research of the Constructing of Cultural Field of Danmu Video Website Bilibili in China. Science Innovation. Vol. 6, No. 3, 2018, pp. 164-176. doi: 10.11648/j.si.20180603.18

Received: April 10, 2018; Accepted: May 14, 2018; Published: June 26, 2018

\begin{abstract}
Danmu is specially defined as all those comments that are inserted on top of the video feeds, which are over-laid on the screen and flow from the right to the left end.This research use the field theory of Pierre Bourdieu as a theoretical framework which combines some other socialogical theories such as theories of Jean Baudrillard to analyze the cultural field of barrage video site in China.I take the website called bilibili as an example to analyze how the field is constructed through three dimension:the website, the barrage video and the users. The Field Theory points out that the complex mutual relationship of all the inner factors lay the basis of a field. As far as this research, the bilibili website provides a cultural production space since it has attracted lots ACG users due to its abundant content resource, strict membership and sophisticated community rules. The exclusive discourse system of barrage video has formed an unique express code system of this field. Danmu language is characterized by repeat, rhetorical question, a set of network language. With these features, danmu language has become an important tool for users to identify people on the same side. Users in the field are the main actors who engage in the cultural production of the field as a team. These three factors work together to construct the culture field of bilibili danmu website.
\end{abstract}

Keywords: Danmu, Danmu Culture, Field Theory

\section{BILIBILI弹幕视频网站的文化场域建构研究}

\section{田阳}

新闻学院, 中国人民大学, 北京, 中国

\section{邮箱}

498378486@qq.com

摘要: 弹 (dàn) 幕指发布在视频上、横穿视频内容, 具备流动性和覆盖性特征的所有评论。本研究以布尔迪厄的场域 理论为框架, 以 以ilibili网站 (简称B站) 为例, 对弹幕视频网站如何构建形成其独特的文化场域加以研究。场域理论指 出, 场域内各要素间的相互关系是建立场域的基础。具体到本研究而言, B站内容丰富, 准入机制严格, 为以ACG文 化爱好者为核心的用户提供了文化生产的场域空间; 以吐槽为核心的弹幕视频排他性的话语体系则构成了该场域的独 特代码; 弹幕族作为场域内的主要行动者, 团结又斗争的参与着弹幕视频网站文化场域的建构。

关键词: 弹幕, 弹幕文化, 场域构建 


\section{1. 弹幕视频网站}

\section{1. “弹幕”的由来和源起}

“弹幕”一词在中日语源中均最早出现在军事领域。中 文中，“弹幕”指炮火射击密集地像幕布一样的炮兵战术 （Barrage）; 日语中，“弾幕(だんまく)”一词则形容防空 炮射击产生的密集碎片。“弹幕”一词由于较为形象地描绘 出子弹密布屏幕的景象, 后该词被引入街机游戏里, 成为 射击游戏的一种。

弹幕视频最早兴起于日本, 弹幕视频网站的鼻祖是 2006年诞生的niconico视频网站，随后弹幕传入中国，在 国内热衷于 $\mathrm{ACG}$ 文化的青少年间引起了关注。 $\mathrm{ACG}$ 是 Animations、Comics和Games三个单词的缩写, ACG文化 特指由来自日本的动画、漫画和电子游戏等系列作品所共 同组成的文化圈。

弹幕视频发展的初始阶段是局限在少数ACG文化爱 好者内部的一种较为小众的文化形式, 以日本动漫和相关 内容为主, 之后随着弹幕这种视频形式被越来越多的人所 认识和接受, 弹幕视频的影响范围日益扩大。我国第一家 成立的弹幕视频网站是2007年建站的AcFun（以下简称A 站）。A站建立之初是单纯的动画连载网站, 后于 2008 年 实现了弹幕播放技术, 开始转型成为全弹幕视频网站。目 前我国国内最大的弹幕视频网站则是2010年成立的bilibili 弹幕视频网站（以下简称B站）。除了 ACG相关内容，普 通的影视剧、音乐、综艺和原创视频等类型多样的内容都 变成了弹幕视频的重要组成部分。相应地, 弹幕视频的用 户队伍也不断扩大, 除了二次元用户, 越来越多的普通观 众开始加入进来，变成弹幕视频的忠实拥歪。

本研究中的“弹幕”特指在弹幕视频网站中, 用户将评 论内容通过弹幕播放器逐条发送到视频画面上, 并随视频 播放再现的新型评论方式。用户用弹幕记录观看视频时的 瞬间感受, 弹幕内容多与视频内容密切相关。这些评论即 时显示并存储在视频中, 并随着视频画面的播放而反复出 现, 形成了一道字幕墙。因此, 本文中的弹幕特指定义为: “发布在视频内容上、横穿视频内容，具备覆盖性和流动 性特征的所有评论”。

\section{2. 选题意义}

弹幕为观众带来了一种新的交互式体验。在观看弹幕 视频的过程中，观众能够即时发表评论、参与互动交流， 体验一种“部落化”的观影模式(周舟,2015)。弹幕视频相较 于传统的视频形式而言, 交互性更强, 受众参与生产和消 费的主动性显著提高。国内的弹幕使用族群聚集在A站、 $\mathrm{B}$ 站等弹幕视频网站, 在参与弹幕生产和传播的过程中形 成了一套约定俗成的排他性“弹幕用语”，进而形成了其独 特的弹幕文化场域。

从理论角度来说, 学术界目前对弹幕的研究多数只是 针对弹幕传播的某一特点展开, 对“弹幕文化”的梳理和探 究具有较大的研究拓展空间。本文以布尔迪厄的场域理论 为依托, 把弹幕文化作为一个独特的文化场域进行分析, 从弹幕视频网站、弹幕文本和弹幕使用族群三个层面对弹
幕文化场域的建构进行整体性的研究，拓展了该理论的应 用对象范围和对弹幕文化的研究视角, 使对弹幕文化的研 究更加系统。通过本文研究证明场域理论在信息时代新媒 体环境下的弹幕视频网站上依然适用, B站表现出场域的 自主性、关系性、斗争性等特点, 且该场域中的行动者 (弹 幕族）显现出与该场域相吻合的惯习。

从实践角度来说，弹幕的影响力已从最初的“二次元” （注：二次元指二维空间, 架空世界, 泛指ACG用户感兴 趣的内容) 走向“三次元” (注: 现实世界), 从弹幕电影、 弹幕晚会到直播平台中弹幕刷屏，（衍生出了“弹幕+”的 文化形式, 弹幕+电影/广告/课堂）人们对弹幕的观感已从 “影响视频观看”逐渐转变为将其视为一种重要的交流手 段。对弹幕文化的研究可以帮助人们更好地认识弹幕这一 新的传播形式以及弹幕族这一新的用户群体, 并对弹幕背 后的传播机制、情感依托和审美情趣有所把握。

\section{3. 核心概念介绍}

\subsection{1. 弹幕视频网站}

本文中所提到的 "弹幕视频网站 " 有如下特征：1)具 有较强的专业性和特定的受众目标, 内容上注重ACG文化。 2) 以弹幕功能为网站的核心功能, 用户互动高度活跃。在 $\mathrm{B}$ 站上, 每个正式会员平均发弹幕数为 247 个; 3)拥有庞大 且活跃的UP主生态圈。网站的生存和发展都以此为依附 和基础。

\subsection{2. 弹幕使用族群}

本文所指的弹幕使用族群是指基于特定弹幕视频网 站、接受或喜爱弹幕评论形式, 并参与弹幕的生产、传播、 消费过程的弹幕用户。

\subsection{3. 弹幕文化场域}

弹幕文化场域指在弹幕视频网站文字和屏幕相结合 而成的文化空间基础上，包括弹幕视频的生产、传播、消 费等各个阶段及弹幕族在此过程中参与从事的所有文化 行为的总和。

\section{4. 文献综述}

\subsection{1. 国内外场域理论研究现状}

“场域理论”的提出者是法国的社会学大师布尔迪厄。 西方学术界尤其是英美国家早在上世纪70年代就已开始 关注布尔迪厄的思想。具体到场域理论而言, 布尔迪厄在 社会学研究中开创性地引入了物理学中“场”的概念。(布尔 迪厄，1993:27)提出，场域可被定义为“在各种位置之间存 在的客观关系的一个网络 (network), 或一个构型 (configuration)”。如布尔迪厄所言, 在现代社会的大背景 下, 由于不同的社会分工而产生了许多不同的小社会, 这 些小社会都有自身的规则和逻辑, 而这些逻辑和规则的变 动会使场域的力量和界限发生变化, 进而促进社会运转。

几乎所有关于布尔迪厄的著作都离不开他的场域理 论。布尔迪厄试图将场域理论作为一种普世方法论, 将其 延伸到社会各个领域, 如政治场域、宗教场域、法律场域、 
美学场域、艺术场域、文化场域。每个场域中都以一个市 场做纽带, 将场域里象征性商品的生产者和消费者联系起 来。后来, 布尔迪厄在《文化资本与社会炼金术: 布尔迪 厄访谈录》中补充提出了资本(capital)的概念: “在场域中 活跃的力量是那些用来定义各种“资本”的东西(布尔迪厄, 1997, 转引自罗德尼·本森, 2003)。”

关于布尔迪厄的场域理念, 不同学者的解读各不相同。 但学者们各异的解读中包含了几个相同点: 第一, 每个场 域中的要素间都存在复杂的关联性, 这种相互关系形成了 场域的基础。第二，同一场域内的不同行动者之间及不同 的场域之间都共同受到元场(政治场、经济场)的限制，不 同的行动者和场域在元场影响下既是独立存在的个体，同 时又可以互相关联。第三，竞争关系存在于各个独立的场 域中和不同场域之间，这种竞争被称为场域的斗争性。场 域理论体现了行动者努力的方向和成果。

学者们对布尔迪厄“场域理论”的延伸研究中对本文 有贡献的主要在以下几方面。其一是关于话语权的研究。 葛兰西在著作《狱中札记》里提到: “社会集团的领导作 用表现在两种形式中——在统治的形式中和以“精神和道 德领导”的形式中, 前一形式体现为上层建筑的国家机器, 后一形式则表现为文化领导权或曰话语权”(葛兰西，1959， 转引自王雨辰, 2001)。法国著名哲学家福柯则认为, “人 和世界的关系是一种话语关系(福柯, 1970, 转引自刘晗,

2010)。”话语是所有认知的来源和动力, 社会团体通过话 语来获得立足之处, 体现其地位和社会关系。由此, 掌握 了话语权就代表着掌握了传播主流意识形态和价值观的 主导权。

其二是对布尔迪厄文化理论的研究。场域理论对社会 文化的形成动因提供了其独特的视野, 它透视了语言文化 背后的符号意义，再现了“符号”间的权力斗争。内蒙古师 范大学常宝的《从布迪厄的“文化资本”理论谈族群文化的 发展问题》一文对族群文化资本的存在形式、“场域”和“惯 习”和族群文化的关系以及族群文化资本的再生产进行了 分析, 论证了族群文化存在的合理性, 为本文对弹幕族的 分析提供了参考(常宝,2011)。新疆大学曹红霞的论文《趣 味与身份认同——布尔迪厄的文化理论》则从“趣味”和 “身份认同”这两个具体概念切入分析, 分析了趣味作为一 种符号在群体间的区隔权力(曹红霞,2011)。这些对布尔迪 厄文化理论的研究为笔者深入了解场域的表达代码系统 (本文中弹幕的话语体系)和探究场域斗争性背后的深层原 因提供了参考。

第三则是网络背景下场域理论的研究现状。网络技术 的发展促使一批新媒体应运而生, 新媒体传播呈现出社群 化、草根性、双向互动性等特点。传统场域中(如政治场 域)曾经集中的权力被下放, 社会秩序趋向平等。英国政 治学家蒂姆·乔丹1999年提出了“网络权力”的核心概念。他 认为, “网络权力是组织网络空间与因特网上的文化与政 治的权力形式(蒂姆·乔丹, 转引自曾玉梅, 2010)。”随着 科学不断发展, 新的媒介形式层出不穷且迭代迅速, 新旧 媒体的关联与影响成了学者们的研究热点。学者们开始讨 论是否能用传统的媒介理论研究心得新的媒介形式的特 征、效果及传播规律。如学者们运用使用与满足理论、知 沟理论、“沉默的螺旋”理论等研究微博、微信、推特等,
关注新的媒介形式对受众心理及社交层面的效果与影响。 有学者认为, 互联网颠覆了传统媒介的传播机制, 传统的 媒介权力被改变, “无组织的组织力量”成为网络媒介权力 的特征（夏德元,2013）。何双秋等人则认为, 新技术的 产生和发展使得新媒体时代权利场域博弯的局势发生了 变化。他们认为在媒介多元化的现在, 受众增强了对媒介 的控制力, 不再以传统媒体作为了解世界的唯一窗口, 传 统媒介的垄断权力被消解（何双秋,2015)。而对场域理 论在网络环境下的直接研究则较少, 局限在微博等新媒体 范围的个案研究。陈联俊的《网络社会“微博”舆论场域的 生成与引导》探讨了微博舆论场域的生成基础、生成机制 与特征, 以及引导路径(陈联俊,2012); 杨金风的《新媒介 时代大众文化身份确认--以布迪厄场域理论分析络文化的 建构》则聚焦新媒体背景下大众文化的场域逻辑和身份构 建是如何实现的(杨金凤,2010)。这些研究为场域理论在网 络时代依然适用提供了佐证。

\subsection{2. 国内外弹幕研究现状}

弹幕作为起源于日本兴盛于中国的独特文化现象, 英 文文献中对其研究内容较少, 而国内从2014年开始, 关于 弹幕的研究文献开始增多。研究者们对弹幕视频和弹幕文 化的研究多是从某一个角度切入。从研究视角来看, 可分 为以下几类:

(1)聚焦于弹幕的传播特和媒介功能。Lili Liu等人认 为, 弹幕作为一种新的媒介形式在传输速度、重申能力、 再加工等方面的媒介功能增强了用户的分享互动意愿和 使用黏性，对用户的感知交互性提升有积极影响(Lili Liu, Ayoung Suh, Christian Wagner,2016)。Zechen Wu的文章同 样肯定了弹幕的二次加工功能影响了用户的参与积极性, 并通过对弹幕文本的抓取和数据分析得出结论称弹幕的 情绪性色彩和视频的受欢迎程度之间存在正相关关系 (Zechen Wu,Eisuke.2014)。日本研究者中村聪史等人则认 为弹幕的“社交注释”功能能帮用户评价视频的优劣, 并证 明弹幕的密集程度和视频内容的趣味性、关键性正相关, 弹幕会显著提升用户观看视频的快感 (Nakamura S, Shimizu M, Tanaka K,2008)。

(2)聚焦弹幕的语言风格。台湾东海大学的Chi-hua Hsiao从礼貌原则和面子理论出发, 分析了弹幕网站的用 户如何在弹幕中操纵话语结构来吐槽。该文章为本研究分 析弹幕的话语体系方面提供了参考, 弹幕族对通过对语言 的创造和共享来展示自己在圈子里的“合群性”, 进而达到 形成群体认同、维护社区稳定性的效果 (Chi-hua Hsiao,2015:109-132)。

(3)聚焦于弹幕的使用族群心理。Yuxiang Zhao等人 (Yuxiang Zhao,Jian Tang,2016)基于史蒂芬森大众传播游 戏理论视角对弹幕用户的心理特征进行分析, 他们认为弹 幕使用者对弹幕本身传播形式的需求大于对弹幕内容的 需求, 弹幕展现了人际传播中的闲聊关系, 对游戏理论“作 为玩乐的传播”这一阐释提供了新的支撑。与之研究视角 相似的一篇文章则将弹幕行为视为一种观看视频过程中 的群体性互动行为, 量化分析了 212 名弹幕用户在使用弹 幕过程中的情感反应和认知状态(Hamasaki M,Takeda H,Hope T,2009)。中国人民大学丁依宁的文章《受众的表 
演与想象：弹幕使用族群研究》则在尼古拉斯的观展/表 演范式的理论基础上, 分析了弹幕族的弹幕生产消费活动 和心理动因(丁依宁,2015)。文章认为弹幕构建了用户间 “观赏共同体”的想象和社群归属感, 对本研究分析弹幕族 提供了借鉴。

(4)将弹幕文化视为一种青年亚文化加以研究。研究中 对弹幕文化进行系统性分析的文献较少, 主要是将其与 ACG文化、日本宅文化或迷群文化相联系, 将弹幕文化作 为一种青年网络亚文化加以关照。Xixian Peng等人分析了 日本酷文化和ACG文化特性对用户使用弹幕的影响, 为将 弹幕文化视为一种青年人网络亚文化的研究角度提供了 参考(Xixian Peng,Yuxiang Chris Zhao,Hock Hai Teo.2016)。 陈一、曹圣琪等人的《透视弹幕网站与弹幕族: 一个青年 亚文化的视角》一文则将弹幕网站视为一个“小众狂欢乐 园”，通过严格的会员机制和丰富活动来增强用户的群体 认同，而把弹幕视作对视频文本的“再加工”。文章提出了 弹幕对文本的 “二次创作”作用, 但忽略了原创的吐槽型弹 幕 (陈一,曹圣琪,王肜.2013)。

在学术期刊和论文之外, B站贴吧、知乎网站、天涯 论坛等网络平台中散落着更多有关弹幕视频网站和弹幕 文化的讨论, 虽然这些讨论涉及范围广泛, 但普通的网络 发言大多停留在现象描述, 缺乏条理性和系统的整合, 专 业角度的解读不足。整体而言, 对弹幕及弹幕视频网站的 研究岁有一定数量, 但深入程度较欠缺, 且针对性也相对 不足。学界目前对弹幕文化的研究呈现出或角度单一、或 理论薄弱的现状。

\subsection{3. 场域理论和弹幕的结合}

本文将弹幕文化研究与场域理论结合起来, 进行了弹 幕文化研究视角的创新。笔者以场域理论来分析弹幕文化, 基于以下几点原因:

(1)场域的自主性和关系性

布尔迪厄指出, “从场域的角度思考就是从关系的角 度思考。”(布尔迪厄,1993:46) 社会行动者一旦进入某个 场域, 就必然会使用该场域特有的表达代码, 并表现出和 该场域需求相吻合的行为。在弹幕视频网站中, 传统的传 一受线性关系被打破，弹幕用户集生产者、传播者、消费 者于一身, 形成多元互动模式, 并通过对语言的解构, 语 义的延伸等方式完成弹幕文本的多层级加工和再创作, 形 成专属群体内部风格化的话语体系。

比如, 在弹幕视频中提醒前方有重要内容时, 用户会 发布诸如“前方高能, 非战斗人员请迅速撤离”等弹幕; 以 及用户会用“2333” (表示大笑) 等大量颜文字来抒发心情。 这些脱胎于ACG文化并经过演化的语言符号形成了独特 的弹幕语言体系。

(2)进入壁垒

布尔迪厄指出: “进入壁垒是进入者拥有的资本数量 与结构的要求。也即某种才能与资格的标准, 不合乎标准 的参与者会被排除在场域之外。每种场域都有或多或少的 制度化标准, 以及一些潜在的、不易察觉的隐藏标准(布 尔迪厄,1995,转引自高宣扬,2004:36)。”

弹幕视频网站中就存在着这样的进入壁垒。B站有着 严格的准入机制。早期 $\mathrm{B}$ 站采取限制注册，只在特定节日
开放的会员制度。2013年5月20日起，B站向全体用户开放 注册, 未注册的普通游客可以看视频但不能发弹幕, 注册 成为“注册会员”后才有权发送弹幕, 但注册会员的权限依 然有限。由注册会员升级为正式会员, 需要由其他正式会 员用硬币购买邀请码的形式邀请, 或者通过 $\mathrm{B}$ 站的答题测 试, 限时一小时内回答 100 道题 (每题 1 分, 60 分及格)。 由于题量大, 范围广, 且题目具有随机性, 非ACG爱好者 很难通过考试。

由这样的进入壁垒所限, B站形成了不同层级的用户, 由高到低依次为: UP主[1], 正式会员, 注册会员, 游客。 不同层级的用户对ACG文化的了解程度不同, 所拥有的权 限也不同, 并因在场域中所处的地位不同而进行有差异的 传播活动。比如, UP主和正式会员可以凭借积分和权限 发送高级弹幕来凸显存在感。高级弹幕分为特殊弹幕和代 码弹幕, 唯有正式会员在使用一定“虚拟资费”、并征得视 频发布者同意后方可发送。高级弹幕可设置文字的移动路 径、常用属性和“时间轴”, 通常被用于制作字幕或歌词(丁 依宁，2015)。

(3)场域的斗争性

布尔迪厄指出，场域具有斗争性：“作为一种场域的 一般社会空间, 一方面是一种力量的场域, 而这些力量是 参与到场域中的行动者所必须具备的; 另一方面, 它又是 斗争的场域; 就是在这种斗争场域中所有的行动者相互遭 遇, 而且, 他们依据在力的场域结构中所占据的不同地位 而使用不同的斗争手段，并具有不同的斗争目的(布尔迪 厄,1999,转引自李全生, 2002)。”

弹幕使用族群在弹幕视频网站中通过观看弹幕, 参与 弹幕生产与传播等行为获得仪式感和社区认同感, 形成 “观赏共同体”。但正如前文所说, 用户因在场域中所处的 地位不同而进行有差异的传播活动。在弹幕用户的群体内 部, 会由于年龄、知识结构和权限的差异而造成分裂。上 传或原创视频的UP主会因为内容好坏而受到其他弹幕用 户的“赞” (投硬币、分享、收藏等形式) 或“弹”(弹幕吐槽, 私信攻击等形式); 会员与非会员之间也会就弹幕质量等 问题出现“掐架”行为。因此, 在弹幕的文化场域中存在着 斗争性, 弹幕用户的“共同体”实际上是松散而脆弱的。

基于以上原因, 笔者有理由认为, 弹幕视频网站的弹 幕文化已形成其独特的文化场域, 对弹幕视频网站形成的 弹幕文化进行整体性关照和分析是合适的。

\section{5. 研究对象和问题}

基于以上文献综述和理论介绍, 本文决定主要使用场 域理论对中国弹幕视频网站的文化场域进行系统性分析, 拟将弹幕视频网站(场域空间)、弹幕视频文本(表达代码) 和弹幕族(场域行动)作为研究弹幕文化场域的三个层次。

$\mathrm{B}$ 站作为国内影响力最大的弹幕视频网站, 本文选取 其作为限定的场域空间, 研究其自主性和独特性。

对弹幕视频文本的研究则主要集中在弹幕的种类、语 言体系和风格等方面。

[1]UP主指弹幕视频网站对上传发布视频用户的统称，也被称为阿婆主 （谐音）、UP（简称）等。UP主具备一定的技术基础、创新思维和原创 能力, 会编辑制作视频, 并以此获得观众的喜爱 
对弹幕族的研究主要集中在研究弹幕使用族群的行 为模式和心理动因上。

研究问题: 中国弹幕视频网站(B站)的文化场域是如 何建构的?

\section{6. 研究方法}

本文的研究对象是基于弹幕视频网站形成的弹幕文 化场域, 因此首先要对本文选定的场域空间—B站的特 色进行分析; 随后, 弹幕视频中最直接的文本表达就是弹 幕语言, 通过对弹幕话语体系的分析我们可直观地感受弹 幕的特征和风格; 另外, 研究弹幕文化场必须深入弹幕族 这个文化群体, 了解他们作为如何参与场域的建构, 形成 了怎样的整体。因此, 本文将用个案研究+深度访谈+问卷 调查(辅助)的方式展开研究。

\subsection{1. 个案研究法}

本文以 $B$ 站为个案进行研究, 对网站的自主特色、B站 弹幕的语言体系和弹幕使用族群特征做全面的考察与分析, 通过个案研究掲示国内弹幕视频网站形成的独特文化场域。

\subsection{2. 深度访谈法+调查问卷法（辅助）}

本研究主要面向B站用户拟出深访问题, 同时参考非弹 幕使用者的意见，就相关话题与对象进行一对一线上/面对 面访谈, 探究弹幕族的弹幕使用习惯偏好和心理动因。深访 用户的选择: 通过B站私信、知乎、豆瓣、微博、微信朋友 圈等方式, 最终选取了12名深度访谈对象, 并按照他们弹幕 的熟悉程度和使用偏好将其分为旁观者(（直接屏蔽弹幕的 人)、普通用户、资深用户分别进行一对一访谈。

同时本文还结合问卷调查的方法，通过微信朋友圈、 微博、 $\mathrm{B}$ 站贴吧等渠道发放问卷, 调查B站用户的行为性 特征, 使对弹幕族的研究获得数量更大的参考样本, 并对 深访起到辅助作用。

\section{2. 作为有限生产场域的B站}

\section{1. 弹幕视频网站传入中国}

弹幕视频网站的始祖是日本的niconico动画。niconico 动画成立于 2006 年12月12日, 成立之初该网站无法上传视 频, 只能将Youtube的视频外链到网站中。2007年2月, 鉴 于版权问题, niconico的视频外链功能被Youtube禁止, 后 推出自己的视频分享服务, 深受观众喜爱。

“niconico动画”的爆红启发了给国内的动漫爱好者, 国内成立最早的弹幕视频网站是Acfun（以下简称A站）。 A站成立于2007年, 取意于Anime Comic Fun,即精彩动漫。 其最初是单纯的动画连载网站, 和市面上其他动漫网站区 别不大, 自身定位特色不明显, 2008年开始A站模仿 niconico推出了弹幕功能, 随后人气迅速攀升, $\mathrm{A}$ 站自此开 始转型成为全弹幕视频网站。但由于A站受niconico影响过 深, 对niconico的资源极度依赖, 受到日本网民以保卫知 识产权为由进行干涉并因此停站一段时间。且A站自成立 以来长期面临着管理层动荡、运营混乱、用户界面不友好 等问题, 使得其原有用户逐渐流失。
2010年3月，A站遭遇了严重的“刷屏危机”，大量会员 在弹幕中恶语相向, 事态失控, 导致整个网站的形象大打 折扣, 众多会员出走。当此之时, 原本仅相当于“A站后花 园”存在的Mikufans改版为哔哩哔哩视频网站 (以下简称B 站), 并因稳定的后台和友好的用户界面而逐渐受到欢迎, $\mathrm{A}$ 站出走的会员、UP主全部流向 $\mathrm{B}$ 站。如今 $\mathrm{A}$ 站无论是从 内容丰富度、页面体验、影响力还是商业运营成熟度来说, 都不如后来居上的B站。而二者的内容布局和核心用户群 又存在着相当大范围的重合, 故而本文选取更有代表性的 $\mathrm{B}$ 站作为分析场域。

\section{2. 作为有限生产场域的B站}

(布尔迪厄, 1993:87)将社会看作一系列不同的,由其自 身“游戏规则”支配的半自主场域(例如,政治、经济、宗教、 文化生产的场域,等等)。它们提供各自独特的交换与获得 的机制,在发展过程中逐渐脱离其他场域的限制和影响, 体现出自己固有的本质。

从成立至今, $\mathrm{B}$ 站内容层面发生了三次进阶式变化: 首先, 日本ACG文化进入中国, 由中国 $\mathrm{ACG}$ 社群孵化出了 中国特有的ACG文化; 进而, 在市场和新流量用户群的共 同介入下，B站衍生出了类型更加细分的多元亚文化; 如 今, B站已经由专注于 $A C G$ 内容的垂直社区壮大成为一个 包括7000多种热门的文化圈组成的社区, 有超过 1 亿的活 跃用户，超过 100 万活跃UP主。B站用户投稿视频每天有 数万级, $90 \%$ 是自制或原创视频 $[2]$ 。

\section{表1 B站内容。}

\begin{tabular}{|c|c|}
\hline 板块 & 具体分类 \\
\hline 动画 & $\begin{array}{l}\text { MAD-AMV (指电玩文化、动漫文化、同人文化界别中的二 } \\
\text { 次创作多媒体作品)、MMD-3D (使用MikuMikuDance和其他 } \\
\text { 3D建模类软件制作的视频)、短片.手书·配音、综合 }\end{array}$ \\
\hline 番剧 & $\begin{array}{l}\text { 连载动画、完结动画、国产动画、资讯、官方衍生、新番时 } \\
\text { 间表/新番索引 }\end{array}$ \\
\hline 音乐 & $\begin{array}{l}\text { 原创音乐、翻唱、VOCALOID (Yamaha开发的电子音乐制作 } \\
\text { 语音合成软件。输入音调和歌词, 就可以合成为原为人类声 } \\
\text { 音的歌声)、演奏、三次元音乐 (三次元艺人的音乐作品和相 } \\
\text { 关)、OP/ED/OST (动漫和影视剧等视频的片头曲、片尾曲、 } \\
\text { 插曲、角色曲等全部曲目)、音乐选集。 }\end{array}$ \\
\hline 舞蹈 & $\begin{array}{l}\text { 宅舞、三次元舞蹈、舞蹈教程 } \\
\text { 单机联机、网络斿戏、电子竞技、音游(以音游为基础的游戏, } \\
\text { 如节奏大师)、Mugen(以Mugen引擎为平台制作、或与Mugen }\end{array}$ \\
\hline 游戏 & $\begin{array}{l}\text { 相关的游戏视频, 以格斗类游戏为主的分类)、GMV: 由游戏 } \\
\text { 素材制作的 } M V \text { 视频。以游戏内容或CG为主制作的, 具有一 } \\
\text { 定创作程度的MV类型的视频。 }\end{array}$ \\
\hline 科技 & $\begin{array}{l}\text { 纪录片、野生技术协会(展示了一定能力的制作或演示的视频, } \\
\text { 以教学视频为主的分类); 演讲公开课、军事、数码、机械 }\end{array}$ \\
\hline 生活 & $\begin{array}{l}\text { 搞笑、日常、美食、动物、手工、绘画、运动 } \\
\text { 二次元鬼畜(主要以动漫为素材的鬼畜)、三次元鬼畜(主要是 }\end{array}$ \\
\hline 鬼畜 & $\begin{array}{l}\text { 电影或网络素材为主的鬼畜)、教程演示(一些优秀的鬼畜教 } \\
\text { 程)。 }\end{array}$ \\
\hline $\begin{array}{l}\text { 时尚 } \\
\text { 广告 }\end{array}$ & 美妆、服饰、健身、资讯 \\
\hline 娱乐 & 综艺、明星、Korea相关: 韩国相关的影视、综艺等资讯 \\
\hline 影视 & 电影、电视剧相关资源 \\
\hline
\end{tabular}

[2] 元浦说文 (2016). 《bilibili董事长陈睿：B站是一个包含7000多热 门文化圈的社区》. 检索于https://sanwen8. cn/p/43f6osg. htm1 
布尔迪厄将文化生产场域划分为两个部分:有限生产 (restricted)的场域(为其他生产者进行生产)和规模生产的 场域(为一般受众生产)。在有限生产的场域中, 生产是为 了其它生产者即场域中的行动者和制度 (布尔迪 厄,1993:132)。对B站而言, 其诞生受益于niconico和Acfun, 以服务于二次元用户起家, 以ACG文化为基石, 虽然随着 开放注册和商业化运营用户规模不断扩大, 但仍然是一个 以服务弹幕爱好者为主的有限生产场域, 并在不断壮大的 过程中形成了自身独特的风格和吸引力。

\subsection{1. 丰富的视频内容资源集聚人气}

$\mathrm{B}$ 站被用户称为 “万能的B站”,寓意不论你想看什么内 容, 都能在 $\mathrm{B}$ 站找到相关资源。

$\mathrm{B}$ 站在动画番剧领域得天独厚。植根于 ACG文化的B 站发迹之初最火爆的内容是动画、番剧和游戏领域。大量 的二次元用户被B站独特丰富的 $A C G$ 内容吸引至此。B站 是国内最积极的日本新番动漫版权购买方。在 $\mathrm{B}$ 站, 用户 可以及时看到带字幕高画质的原番日漫。在B站追番长达 5 年的资深用户 $\mathrm{B} 0$ 然在访谈中表示： “最早大家都是因为 $\mathrm{B}$ 站有很多番啊日漫啊 (才来B站) 追, 像齐木楠雄啊、灵能 $100 \%$ [3] 之类，然后 (ACG) 用户越来越多，口碑效应慢慢发 酵, B站才逐渐变成一个二次元聚集地, 又慢慢发展成今 天这样。”2016年, B站引进了113部日本新番动漫, 继续 领跑国内视频网站。

B站还对国产动漫进行大力支持和培育。2015年起, $\mathrm{B}$ 站开始系统推广包括上海美术电影制片厂、央视出品的 多部正版国产经典动画作品比如《黑猫警长》、《大闹天 宫》等, 这些作品的平均点击量在 20 万左右, 弹幕评论超 过 1500 条，众多90后、00后用户表示在这里找回了童年。 同年12月是 $\mathrm{B}$ 站与新浪微博联合打造国产动画月, 发动年 轻人一起讨论“安利国产动画的 100 种方法”等话题 [4]。此 外, $\mathrm{B}$ 站还支持动画作品的商业化, 其参与推广、投资的 多部作品如《大圣归来》等, 在用户中收获极佳口碑。

B站UP主“直真真”在“你认为 B站靠什么能力在商业 竞争中胜出”问题下回答称, B站吸引自己的最重要原因是 资源优势。

直真青直: 我不知道国内视频网站具体的版权情况是怎 样，从我自身而言的话，一般我想追什么番我都会先在B 站找资源，B站找不到再去别的网站找。而且B站不只是 动画资源丰富, 其他各种类型资源都很多, 比如有冷门电 影合集啊，还有同步更新的一些日剧韩剧。其实不是我不 想看正版内容, 而是买都没有地方买。后来发现在 $\mathrm{B}$ 站, 无论是国外纪录片、综艺还是文艺片都有up主投稿, 而且 画质感人, 我不在这看在哪看。

\subsection{2. 严格的进入壁垒凝聚核心用户群}

布尔迪厄指出, 每个场域都有其独特的进入壁垒。进 入壁垒是进入者拥有的资本数量与结构的要求, 也即某种 才能与资格的标准, 不合乎标准的参与者会被排除在场域

[3]《齐木楠雄的灾难》和《灵能百分百》均为日本动画 [4] 中国动漫产业网 (2015). 《B站联合新浪微博打造12月国产动画月》 检索于http://comic. k618. cn/wztt/201512/t20151215_6376913. htm
之外。“每种场域都有或多或少的制度化标准，以及一些 潜在的、不易察觉的隐藏标准(布尔迪厄,1993:79)。”对弹 幕视频网站的场域而言，其进入壁垒为其严格的准入机制 和完善的社区规则。

(1)准入机制

$\mathrm{B}$ 站目前的会员级别分为 6 级。不同等级的会员拥有不 同的社区特权, 等级的提升则通过网站活跃度实现, 最高 是6级。2014年B站开放注册后, 只要注册就能成为“注册 会员”，但注册会员权限很少，不能发特色弹幕，也不能 观看一些只向正式会员提供的视频资源。从注册会员成为 正式会员则需要通过一整套答题测试, 通过答题, 可以成 为 1 级会员。 1 级会员只能发最简单的滚动弹幕, 2 级会员 可以发顶部和彩色弹幕, 到达3级则可以发底部弹幕(即做 字幕君), 只有正式会员才能上传视频(成为UP主)。B站会 员的具体级别权限见下表。

表2 会员权限。

\begin{tabular}{llllllll}
\hline 特权与等级 & LV0 & LV1 & LV2 & LV3 & LV4 & LV5 & LV6 \\
\hline 滚动弹幕 & X & $\sqrt{ }$ & $\sqrt{ }$ & $\sqrt{ }$ & $\sqrt{ }$ & $\sqrt{ }$ & $\sqrt{ }$ \\
彩色弹幕 & X & X & $\sqrt{ }$ & $\sqrt{ }$ & $\sqrt{ }$ & $\sqrt{ }$ & $\sqrt{ }$ \\
高级弹幕 & X & X & $\sqrt{ }$ & $\sqrt{ }$ & $\sqrt{ }$ & $\sqrt{ }$ & $\sqrt{ }$ \\
顶部弹幕 & X & X & $\sqrt{ }$ & $\sqrt{ }$ & $\sqrt{ }$ & $\sqrt{ }$ & $\sqrt{ }$ \\
底部弹幕 & X & X & X & $\sqrt{ }$ & $\sqrt{ }$ & $\sqrt{ }$ & $\sqrt{ }$ \\
视频评论 & X & $\sqrt{ }$ & $\sqrt{ }$ & $\sqrt{ }$ & $\sqrt{ }$ & $\sqrt{ }$ & $\sqrt{ }$ \\
添加Tag & X & X & $\sqrt{ }$ & $\sqrt{ }$ & $\sqrt{ }$ & $\sqrt{ }$ & $\sqrt{ }$ \\
视频投稿 & X & X & $\sqrt{ }$ & $\sqrt{ }$ & $\sqrt{ }$ & $\sqrt{ }$ & $\sqrt{ }$ \\
购买邀请码 & X & X & X & X & 1 个/月 & 2 个/月 & 3 个/月 \\
历史播放上限 & 100 & 100 & 120 & 150 & 200 & 200 & 200 \\
\hline
\end{tabular}

成为 $B$ 站正式会员所需的答题测试为一整套试卷, 第 一部分是20道“弹幕礼仪题”, 这些题目是考察用户应该如 何文明发送弹幕的题目, 从题库中随机选取, 旨在提醒答 题者遵守 $\mathrm{B}$ 站的规章制度，保障弹幕质量。如“在观看视频 中遇到违规弹幕时应该?A. 进行举报; B. 立即关机; C. 吃 键盘; D. 自己也发不和谐的弹幕”等。

答完“弹幕礼仪题”后, 答题者还需通过有一定专业难 度的自选题测试。用户可从 $\mathrm{B}$ 站的六大类17小类分区领域 中, 根据自身所长选择3-10个擅长的领域做答。总题量为 100 道, 一小时内答对 60 题即可通过测试, 成为正式会员。 笔者选取自己较为擅长的“影视剧”、“文综”、“娱乐”标签, 不难看出题目设置涉及知识面广、专业性较强, 如: “肕 有阴晴圆缺, 用科学的观点看待这种现象其原因是? ”“金 坷垃的总部在美国？”等。

接触B站一年半, 但至今还没有成为正式会员的“碧 ” 在接受访谈时表示: “我没有没有通过答题测试, 大概只 答了 $20 \%$ (就不想答了), 因为觉得太耗时间, 而且很多题 目也不熟悉。”而仅花了半个多小时就答完全部题目, 以 95分高分成为正式会员的“金簿”则自豪地表示: “我觉得 题目不难啊, 常看动漫, 感觉答题无压力。”他是一名即 将参加毕业的研究生, 接触B站已有三年时间, 几乎每天 都要耗费数小时在 $\mathrm{B}$ 站观看动漫视频。由两位访谈对象的 表达不难看出, B站的答题机制在用户间设置了一道严格 的准入门槛, 将部分缺少热情和耐心, 或对 $\mathrm{B}$ 站内容领域 了解不够的用户排除在正式会员之外。

(2)社区规则 
关于网络社区, 范·戴克认为: “网络社区超越了传统 社区中的时空制约, 但是依然服务于现实社会中社会的、 文化的或者精神方面的普遍兴趣, 从一般的到特殊的兴趣 或行动(范·戴克, 1997, 转引自江根源,2014)。”正如前文所说, B站早期的兴趣基础是 $\mathrm{ACG}$ 文化, 社区成员是 $\mathrm{ACG}$ 迷群; 现在虽然准入限制有所松动, 但热爱 $\mathrm{B}$ 站提供的内容, 热 爱弹幕视频仍是进入这一社区的基本。

相较于传统社区而言, 网络社区规章制度实现难度更 大, 但随着用户群不断扩大, 社区的管理和维护正是凝聚 用户，持续发展的重要因素。B站就有着完整的社区规范 和惩罚机制。比如严禁色情、暴力内容, 遵守国家基本法 律, 严禁发表涉及国籍、种族、宗教、性取向、残障等针 对特定群体或对象人身攻击的语言等[5]。如果出现内容不 当的弹幕, 用户可将其举报给管理人员, 发布该弹幕的用 户会被扣除相应的“节操值”。节操值是B站对用户使用行 为进行管理的一种方式, 低于一定界限会影响社区功能的 使用, 如用户被禁言, 无法发布私信、评论等, 而举报不 良弹幕的人经核实后则会得到节操值增长的奖励, 恶意举 报的用户会导致账号被封停。

\subsection{3. 优质的社区氛围形成群体归属感}

$\mathrm{B}$ 站的用户对于网站有着极高的认同感和忠诚度。比 如在B站“拜年祭”视频中, 很多人会在弹幕中发布“此生无 悔如B站”等言论来表达自己对B站的热爱和群体认同感。 $\mathrm{B}$ 站优质社区氛围的形成, 离不开以下因素:

(1)以ACG文化为基石, 扩展多元文化圈

在中国，也只有 $A 、 B$ 站才专心做 $A C G$ 圈子，对于其 他网站而言 $A C G$ 只是附加板块, 但是我们要的不是捎带, 而是专心致志。所以对我这样的二次元爱好者来说, 看番 的话首推B站。而且大家看番没那么局限, 看番看到喜欢 的角色就会有人做角色MAD, 听动漫歌的又可以延伸到 三次元音乐和宅舞, 游戏更不用说了, 还有鬼畜, 王的哲 学等各种, 初始的氛围形成了, 后来做大了就会形成作者 主动来投稿的良性循环。(未见到的明日之诗,2017)

UP主“小鱼SOLO”则表示: “B站宅文化氛围很浓，而 且各个区内容切割很明确, 各司其职, 不管你喜欢什么都 能在 $\mathrm{B}$ 站上找到相应内容和团体。看韩剧的看韩剧, 动漫 迷追番, 宅男看妹, 追星族看八卦, 萝卜青菜各有所爱, 在B站你总能找自己喜欢的款。”

(2)高清的视频质量和良好的互动反馈机制

在B站看视频，总能在视频开头看到“画质感人”之类 的弹幕。B站追求高清晰度的视频资源, “小鱼SOLO”表示: “跟中国大部分视频网站一比, B站的视频清晰度简直泪流 满面, 很多认真的UP主都会传720P和1080P高清的, 完全 免费不要钱! 比如说我就已经被高清视频惯坏了, 一去土 豆看到那渣画质就想直接右上角（退出）。”

“小鱼SOLO”是影视剧区的一名UP主。丰富的UP主资 源是B站的一大优势, 对UP主来说, 在B站上传视频, 他 们能通过弹幕等形式和网友互动, 在获得更多的肯定和满 足感的同时, 还能得到视频制作技能的提升。

[5] 馒头商学院 (2016). 《B站是怎么做内容、社区和UGC的》. 检索于 http://www. chinaz. com/manage/2016/1101/603921_2. shtm1
直真埴: “我非常喜欢 $B$ 站的弹幕, 因为我每次上传视 频, 除了传统的播放量, 收藏量, $\mathrm{B}$ 站还能显示同时观看 的人数, 而且大家会通过弹幕和硬币给你反馈啊, 或者发 私信互动啥的, 我就能很清楚地看到大家对我的视频是什 么态度, 比如说吐槽的点在哪儿啊, 我剪的一些冷门内容 有没有人get啊, 这些体验对UP主来说很珍贵, 就是感觉 你花心血剪出来的东西是有人在乎的。（而别的网站不能 带给我这样的互动体验。) 之前我有一次在土豆上传了一 个视频, 强行给我在片头插了一分钟广告就不说了, 而且 只能看到播放量和点击量, 沙发 (第一条评论) 空着其他 啥都没有, 也不知道点进来的用户看完了没有, 喜欢吗, 有什么意见和吐槽吗, 一个大写的没劲, 以后就都不再去 了。”

(3)丰富的线上线下活动延伸用户情感纽带

$\mathrm{B}$ 站的线上活动丰富多彩, 这些活动通常根据不同节 日而推陈出新, 以此来吸引使用族群的注意力。比如元旦 的“新年祈愿”活动, 情人节的“踢翻这碗狗粮”等。其中“拜 年祭”和“弹幕大赛”是 $\mathrm{B}$ 站两个最具代表性的线上活动。

从2010年开始, B站上线了其专属的新春贺岁视频“拜 年祭”。“拜年祭”由B站UP主以及哔哩哔哩视频部等创作 分段视频, 内容主要由四部分组成。第一部分为歌舞, 通 常会出现一些“神曲”，比方2015年的《权御全国》，2016 年的《九九八十一》，以及2017年的《万神纪》; 第二部 分则以言语节目为主, 比如2015-2016年动漫中的虚拟偶 像洛天依说相声; 第三部分则集合了UP主们的原创故事, 最后一部分则由音乐区UP主们献上“音乐大独奏”[6], 将 气氛推向高潮。“拜年祭”被誉为年轻人的“二次元春晚”, 传统歌舞换成了宅歌宅舞, “发来贺电”的是一些人气UP 主和鬼畜明星, 春节的传统习俗经过重新包装, 成了年轻 人们的狂欢派对。

弹幕大赛的前身则 $\mathrm{B}$ 站的“蓝白弹幕祭”。该节目由 devel等用户于2011年发起, 旨在展现需要特殊技巧的弹幕 艺术。弹幕艺术制作有三种形式, 分别为普通弹幕、特殊 弹幕和代码弹幕, 后两者需要制作者具备专业的编码知识。 该节目发起后迅速变成 $\mathrm{B}$ 站全民狂欢弹幕节, 以此为基, B站官方于 2012 年举办了第一届“弹幕大赛”, 吸引了很多 对高级弹幕感兴趣的用户参加。2015年第二届弹幕大赛启 动, 分为“弹幕艺术类” (对弹幕进行观感上的设计使其具有 艺术感) 和“游戏体验类”(利用代码使弹幕实现多种功能) 两大类, 在B站引发了一阵弹幕狂潮。

除了线上交流, $\mathrm{B}$ 站还紧贴市场需要, 将二次元用户 聚集到现实生活中, 开发了一系列以动漫为主题的线下活 动。其中最负盛名的活动是 BML (全称 bilibili Marco Link), 该活动开始于 2013 年, 是 $B$ 站每年一届的网友线下大聚会, 现场会邀请一些B站的知名UP主和艺人进行歌舞表演。如 今该活动的规模已经从第一届的 800 人, 发展到最新一届 的数万人次。该活动不仅给 $\mathrm{B}$ 站给 $\mathrm{ACG}$ 用户带来的一场狂 欢, 更将二次元文化推向生活中更广阔的青年群体, 既增 加了既有用户的黏性, 也吸引更多年轻人进入 $\mathrm{B}$ 站。

“线球君”参加了B站2015BML活动, 她表示:

[6]作者不详. 《B站拜年祭是怎样成为二次元春晚的》. 检索于 http://www. techweb. com. cn/prnews/qiyenews/archives/7523. htm1 
以前我就是在 $\mathrm{B}$ 站潜水, 在各区随便逛逛, 也不怎么 发弹幕。但是今年夏天我参加了B站2015BML活动, 那种 感觉真的不一样, 就是一种找到了组织的归属感, 一群志 同道合的人一起聚会, 以前你觉得小众的爱好发现啊原来 有这么多人喜欢, 到活动结尾的时候大家一起拿手电筒组 成星海, 感觉跟看演唱会一样, 我想我会永远记得那种感 觉。

从上文分析可以发现，B站泛二次元化的丰富内容布 局和严格的进入壁垒将该场域和其他场域划分开来, 并发 展过程中展现出其“固有的本质”即自主性。这些有竞争力 的内容、明晰的规则和高凝聚力的社区氛围形成了布尔迪 厄所说的场域边界“合法的定义权”，B站制定了本场域内 专属的规则, 弹幕的发布和弹幕族作为主要行动者在其中 的一切活动都要遵循这些规则。

\section{3. 弹幕视频和弹幕族}

\section{1. 弹幕视频}

在弹幕视频中, 用户间可以进行更积极地互动, 而非 被动接受视频内容。弹幕族通过发送弹幕来抒发情感, 发 表意见, 和处在同一场域中的其他观众产生共鸣, 建立连 结。对用户来说, 视频中的弹幕有时比视频本身更受欢迎。
布尔迪厄指出: “社会行动者一旦进入某个场域，必 然表现出与该场域相符合的行为, 以及使用该场域中特有 的表达代码。”(布尔迪厄,1993:76)这种特有的表达代码， 就是弹幕自成一派的话语体系。对不了解弹幕语言的人而 言, 很难从这种“不明所以”的表达方式中获得愉悦。没有 观看弹幕视频习惯的“皓月当空”在访谈中表示:

我看视频时不会选择开弹幕, 因为我感觉开着有点喧 宾夺主的意思, 分散我看视频的注意力, 而且弹幕多的情 况下 (在屏幕上) 过去的又快, 我根本就看不清他们说了 什么, 看时间长了还眼晕。最重要的是我可能理解不了很 多弹幕的梗吧, 感觉没营养的内容挺多, 有时候看见大片 刷屏的弹幕就不知道他们在刷啥, 有点不明觉厉。(皓月 当空)

参考香农的信息论中所提出的, 弹幕视频相较于传统 的视频而言, 本身就带有一定程度的“噪音”性质, 它所呈 现的信息是混杂式、溢出式的，这也就决定了所谓的熵值、 咒余、噪音具有个体化和相对性。甲之熊掌可能成为乙之 砒霜, 因此, 本文中对弹幕视频的研究主要是对弹幕话语 体系的研究。

\subsection{1. 弹幕类型}

在B站看视频不难发现, 弹幕语言虽然经常让人“不明 觉厉”, 但并非天马行空, 而是有迹可循。笔者对 B站经常 出现的弹幕语言做了简单的类型梳理, 如下表所示。

表3 弹幕类型。

\begin{tabular}{|c|c|c|}
\hline 类型 & 含义 & 典型弹幕示例 \\
\hline 解释说明型 & 涉及到背景、典故、专业名词或翻译时有观看者在弹幕中作出的解释。 & 暂停看红字君科普 \\
\hline 剧透型 & 故意提示剧情走向 & “凶手是XXX” \\
\hline 原创吐槽 & 针对视频中的情节、语言或人物行为或其他弹幕内容进行调㑆、批判等 & 常被用户称为“神吐槽”, 依赖于视频内容产生 \\
\hline 符号语气词 & 用数字、字母、特殊符号或表情文字表达情绪 & $2333 、 666$ \\
\hline 空耳型 & 在弹幕中常见于把一种语言按发音强行用其他语言文字代替。 & $\begin{array}{l}\text { 俺痒痓啊呀呀(原句: “Ah, ja, ja,ja, ja!”出自《元首 } \\
\text { 的愤怒》) }\end{array}$ \\
\hline 引战互撕型 & $\begin{array}{l}\text { 针对各自喜欢的角色人物或有争议性的话题在弹幕里进行争论, 内容可基 } \\
\text { 于或游离于视频内容 }\end{array}$ & $\begin{array}{l}\text { “XX党头顶青天” } \\
\text { “吃狗肉的出门左拐不送” }\end{array}$ \\
\hline 回应型 & $\begin{array}{l}\text { 依赖弹幕语境产生的回应, 对视频内容或视频中其他弹幕作出响应、赞同 } \\
\text { 或计数等行为 }\end{array}$ & 站楼上;前面的别跑 \\
\hline 聊天型 & 多发生于同时观看视频人数较少的时刻 & “四六级没过的小伙伴来聊一聊”; \\
\hline 字幕型 & 用户对国外刚出的新番或生肉视频资源在弹幕中进行翻译 & \\
\hline
\end{tabular}

\subsection{2. 弹幕语言特征}

布尔迪厄认为, 在有限的生产场域中, 供给方同时也 是需求方, 限定的生产场域即自主化的场域, 或者说具有 自主性的场域。他认为, 一个场域越是自主的, 这个场域 的生产者只为本场域其它生产者生产而不为社会场域的 消费者生产的程度越大(布尔迪厄,1993:132)。对弹幕视频 网站来说, 弹幕的表达形式是排他的。弹幕并非点对点的 传播, 而是多对多的扩散, 本质上更像是群体内部的自娱 自乐。弹幕的生产和消费是为了在处在同一场域中的用户 间引起共鸣, 而对于该场域之外的一般受众而言, 在已经 习惯于传统视频提供的 “纯净” 的观看体验前提下, 弹幕这 种形式对他们而言可能只是遮挡画面、分散注意力的乱码, 甚至让他们感到晕眩或烦躁, 而完全体会不到观看弹幕的
乐趣。而要研究这种乐趣, 除了了解弹幕中经常出现的语 言类型, 更需要对弹幕的语言特征加以分析。

(1) 重复性

弹幕中会反复出现相同或类似内容的弹幕。发布弹幕 和观赏弹幕的人通过累积、并置和扩展等重复性刷屏的方 式来对字句含义进行新的解读。用户通过重复刷屏有趣的 弹幕评论以强调自己的群体内身份, 或是为了使弹幕中的 互动朝着他们希望的方向前进。

例: “平平无奇的男子”

这个梗出自电视剧《圆月弯刀》中, 古天乐饰演的丁 鹏一角武艺高强, 打败了某江湖高手后在饭馆吃面, 店中 的三个路人议论道: “看他的样子平平无奇, 没想到武 功......" 鉴于古天乐当时正处在颜值巅峰期, 剧中扮相非 
常俊美, 由于观感和台词错位引发弹幕疯狂吐槽: “长这 么帅叫平平无奇”。

在这个情景下, 网友认为最适宜的弹幕内容是吐槽这 句台词，因此大量重复性的“平平无奇”弹幕变成了网友的 一项重要工具, 既能通过刷屏凸显“我分享了这个梗”这样 一种参与其中的存在感, 又能进一步强化对台词本意的解 构。正如Goodwin指出的, 对字、词、句进行精确的或变 体性的重复能形成一种魔性的节奏模式以吸引受众 (Goodwin,Charle,1986)。

(2) 成套的网络用语

弹幕中网友喜欢用既有或独创的网络用语来表达自 己的情感、思想和态度。比如, 当用户对UP主上传的内 容表示赞叹时，弹幕中会出现“楼主你看我趾的姿势标准 吗”、“献上膝盖”等言论; 当视频中出现让人意想不到的 情节时，弹幕中则会出现“666”、“6到飞起”等评论，表示 被折服和膜拜的情感; 而当视频内容中出现有性意味的内
容时, 弹幕中则被大片“一言不合就开车”等内容刷屏。这 些网络用语在弹幕中屡见不鲜, 弹幕族通过对网络用语的 熟练掌握和合理使用, 来建立弹幕族群中的群体关系。

(3) 吐槽的核心风格

梵·迪克将“风格”定义为: “说话人的社会特征和说话 场合中社会文化的具体特征的显现形式或标志(梵·迪 克,1997,转引自江根源,2014)。”他认为风格是文本的语境 特征。风格由“说话人”对语言形式进行有倾向性的选择和 使用而形成, 选择某种特定的语言形式就会形成相应的的 风格特征。具体到弹幕视频的语境中而言, 弹幕的核心风 格是吐槽, 能否从吐槽中获得观看视频的额外乐趣是检验 用户是否融入了弹幕族的一个重要方法。

在各种弹幕类型中, 最受欢迎的一类是吐槽型弹幕。 据问卷调查结果显示，在“你最喜欢的弹幕类型”和“你为 什么喜欢在 $\mathrm{B}$ 站看视频”两个问题中, 得票最高的答案均与 吐槽相关 (分别为 $80.66 \%$ 和 $42.8 \%$ )。

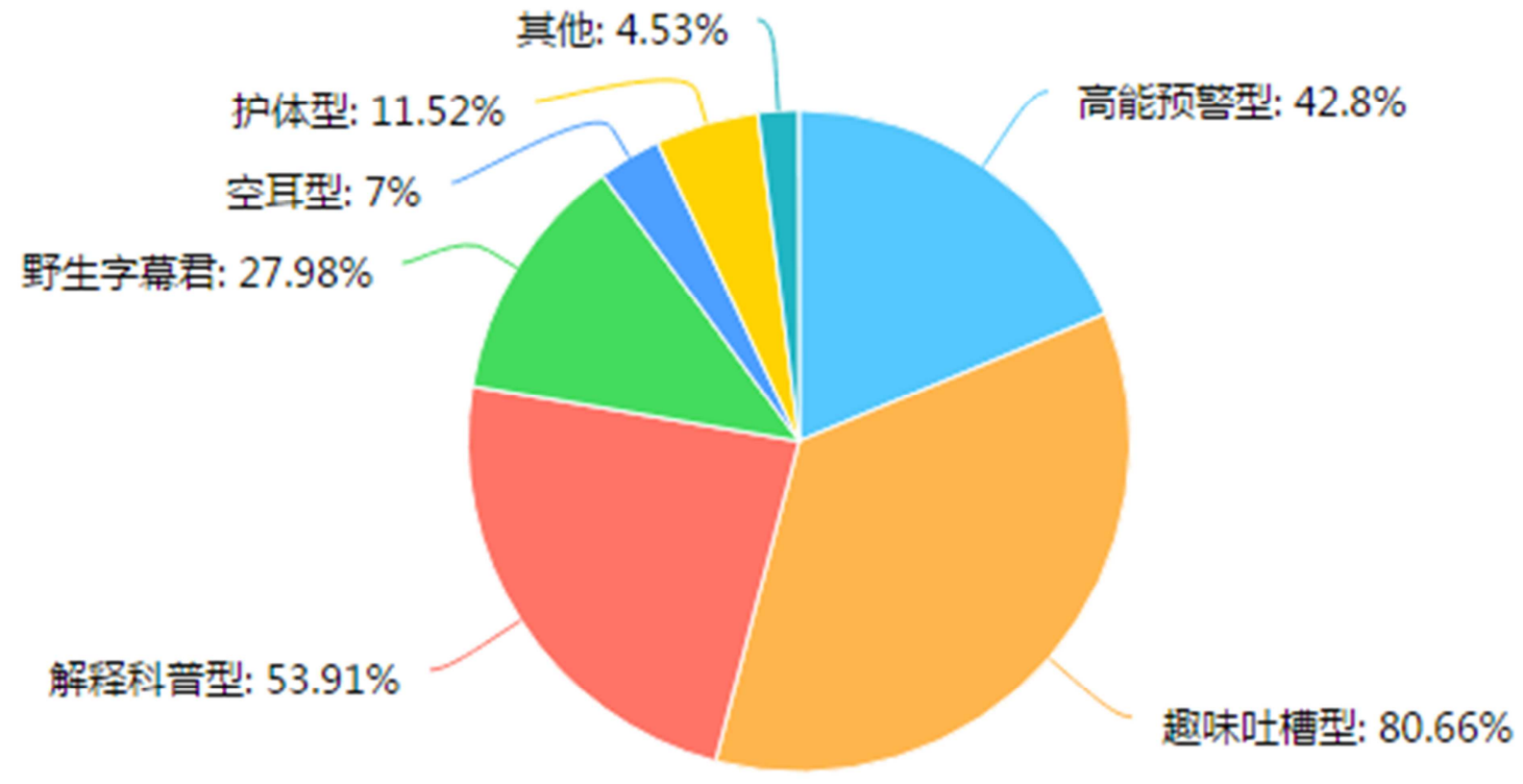

图1 你喜欢的弹幕类型是?

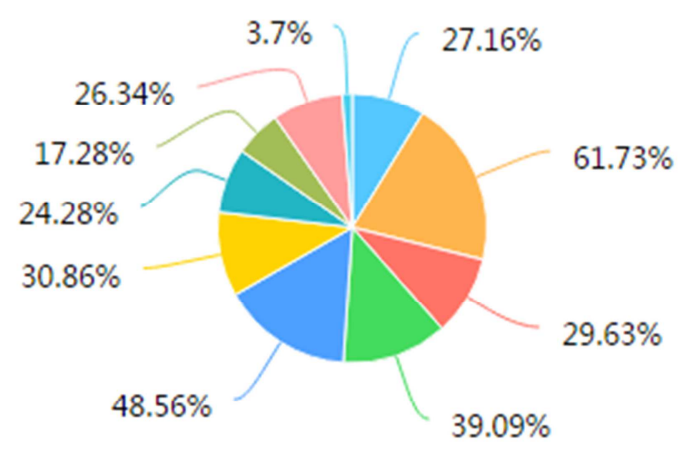

图2 你喜欢在 $\mathrm{B}$ 站观看弹幕视频的原因是? 
用户喜欢吐槽类的弹幕, 其一是弹幕吐槽满足了用户 的互动需求。一条好的吐槽通常简短而犀利, 会即刻引起 观看视频的大多数网友的响应（刷屏）。吐槽的终极目标 是让观看者感到愉快。用户检验吐槽好坏的标准是, 它是 否好笑, 或讽刺了某人, 抑或能引起共情。正如Crystal 所说: “语言只有在这样的情况下才算是成功的: 大家用 戏谑的方式来赞赏彼此的机智”。(Crystal,1998)

天涯论坛的一份题为“ $\mathrm{B}$ 站弹幕有哪些神吐槽”的帖 子, 回复高达 1400 多条, 网友纷纷表示在B站看视频时经 常被弹幕中的神吐槽“笑到不能自理”。比如, 微博用户 “琳酱”正在B站看《三生三世十里桃花》，由于其中的男 主角夜华经常遭受天雷䢃身的惩罚, 每当这种镜头出现 时弹幕就会刷“夜华是用电大户啊”, 琳酱看到会哈哈大 笑。“就觉弹幕怎么这么有才, 这个梗出现后我们就经常 刷“查电表的上门了’之类的弹幕, 只有在 $\mathrm{B}$ 站追剧的人才 能看懂。”

知乎网友谢坤视频本身的信息量巨大, 细节丰富, 是这种错位幽默感最好的载体。“弹幕与其说是满足聊天 的、社交的需求, 不如说满足的是用户吐槽的需要, 弹 幕吐槽的本质我觉得是利用信息的错位营造一种幽默的 氛围。”

另外, 弹幕中的吐槽有时还会形成竞争关系, 即有人 发了一条精彩的吐槽, 后面发弹幕的用户会想方设法发一 条更好笑的或者角度更独特的以示回应。在弹幕中, 匿名 性让大家不用考虑彼此的颜面, 吐槽的言辞越犀利, 其在 建立群体互动性中的作用反而更能得到彰显, 而线下人际
交往中那种为了保护颜面的, 有分寸的礼貌性言辞则完全 被弹幕语言所抛弃。

\section{2. 弹幕使用族群}

霍尔在《通过仪式抵抗》中提到这样一种青年群体: 他 们的身份在特定的历史时刻得以显露和确认, 并被（自己或 别人) 贴上标签, 如光头党、摇滚派、泰迪男孩或摩登族等。 “弹幕族”正是一个这样的群体。他们作为弹幕视频网站文化 场域的主要行动者和建构者, 形成了群体独特的风格。

\subsection{1. 弹幕族的人口统计学特征}

为了解弹幕用户的基本情况, 笔者在B站贴吧发布问 卷主题帖, 同时通过微信朋友圈、微博超级话题、知乎平 台进行问卷发布。本次问卷通过问卷星平台制作并发放, 截至2017年3月1日，共收回有效问卷356份，弹幕用户的 人口统计学特征结果如下。

根据问卷结果显示，参与问卷的对象中超过 $70 \%$ 的B 站用户年龄在 28 岁以下, 女性数量是男性的两倍以上, 学 历以大专以上为主, 呈现出年轻化、高学历、女性占主导 的趋势。据B站官方统计, B站全体用户中 $0-17$ 岁的用户 占 $37.55 \% ， 18-24$ 岁的用户占 $29.96 \%, 25$ 岁以上的用户加 起来不到 $10 \%$ 。青少年和大学生群体是B站用户中绝对的 主流。本问卷由于渠道等方面因素, 较难获取18岁以下用 户样本, 但用户主要年龄层符合B站官方发布的年龄范围。

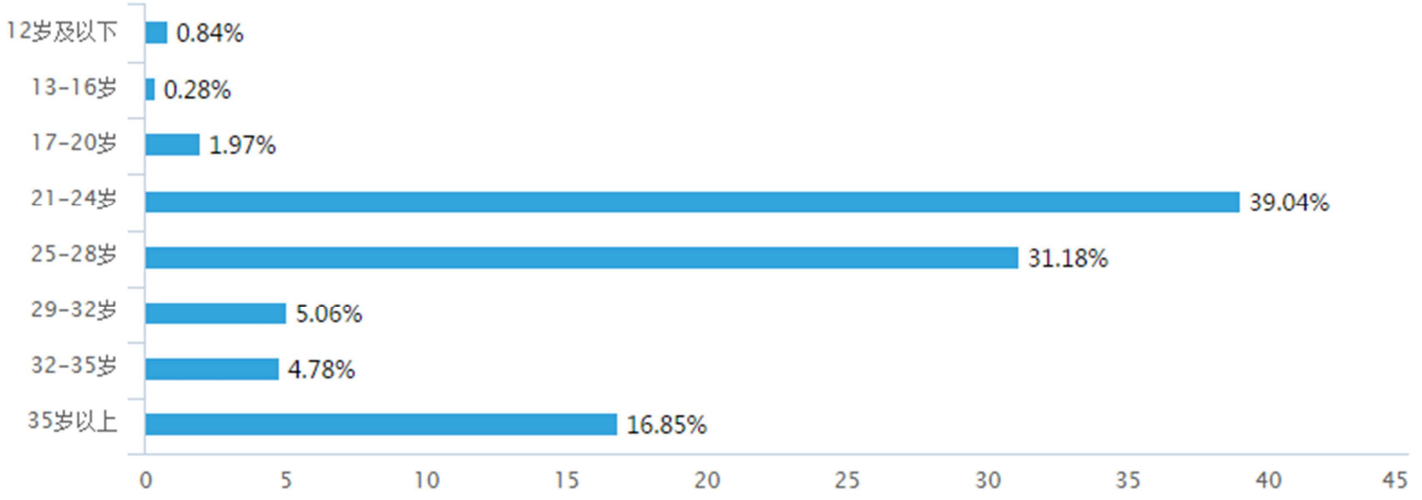

图3 用户年龄。

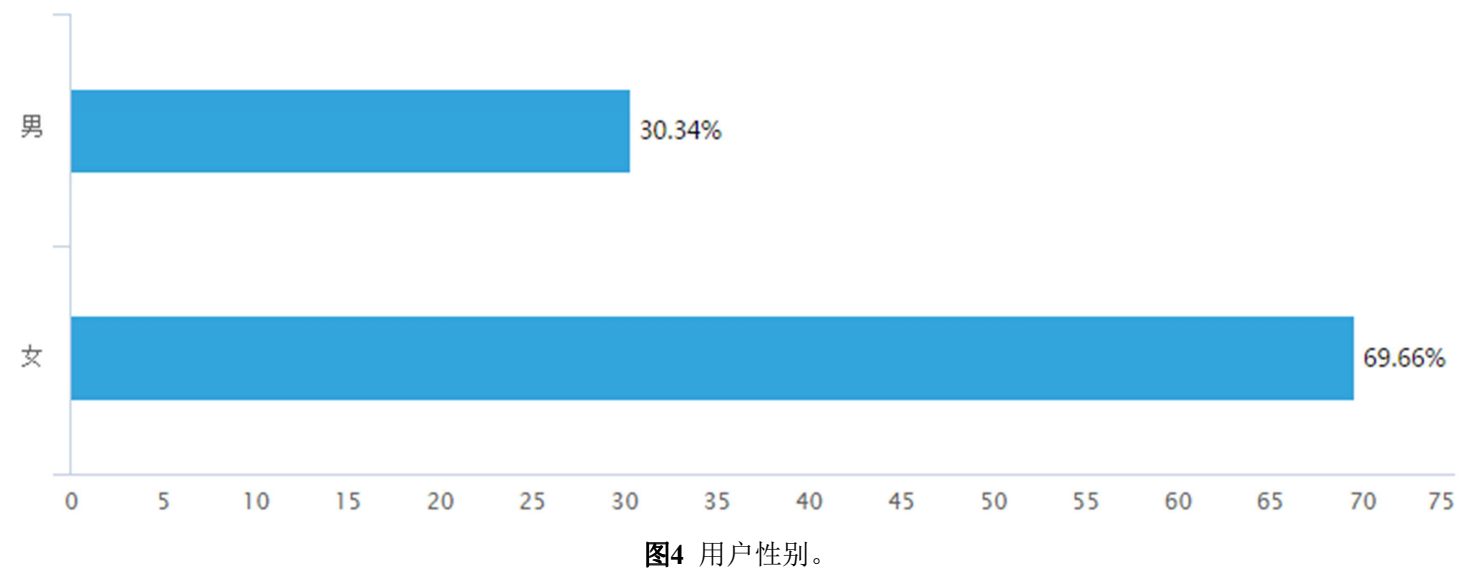




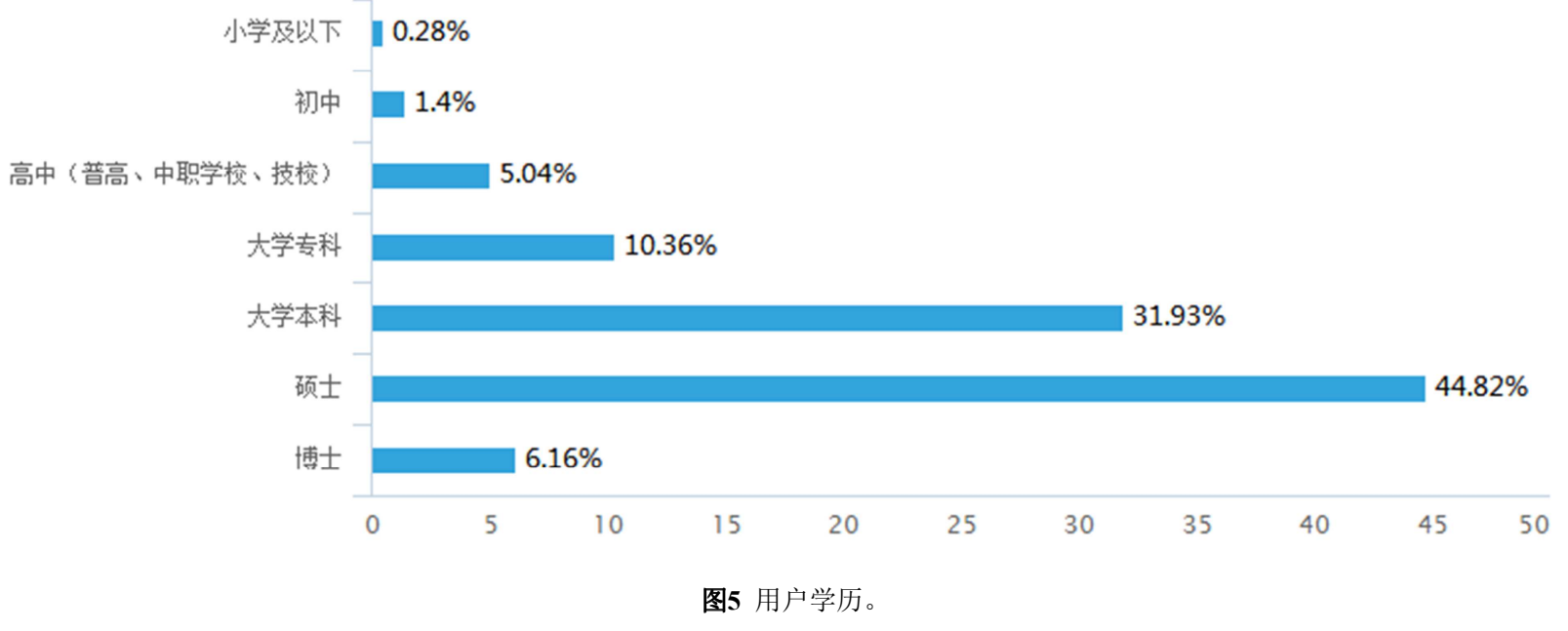

\subsection{2. 弹幕用户行为角色的模糊}

ABercromBie和Longhurst[7]将受众按照涉入程度分 为五个层级, 依次为: 无特定媒介使用习惯的普通消费者; 受到某种特定类型文本吸引，但彼此之间没有接触的迷群; 针对特定文本、大量使用媒介而且相互联系、形成非正式 的网络联结的“崇拜者(cultures)”; 组成严谨、专注的特定 文本，有一定组织基础的“狂热者（enthusiast）”; 由狂热 者发展出来的有专业生产能力的小规模生产者（petty production）。受众按照涉入程度, 层级越高者投入的时 间与精力越多, 同时对文本的解读能力也越专业。

对弹幕使用族群而言, “UP主”和高级弹幕制作者等或 许处于“狂热者”到“小规模生产者”之间，而大部分用户则 处于“迷群”与“崇拜者”范畴之内。从场与理论视角而言, 布尔迪厄认为, 对特定的文化产品而言,生产者和受众之 间存在着基本的同源性(homology)。由此,存在一个“在两 个利益体系(生产和接受)之间预设的和谐...... 一方只需对 的依者进行布道 (布尔迪厄,1993:156)。”而在弹幕视频网 站的场域中，由于弹幕族行为角色的模糊，这种“同源性” 变得更加明显。用户可以在视频生产者（UP主）、视频 消费者、弹幕生产者、弹幕消费者四种角色间相互转化, 形成一种“所有人对所有人”的互动模式，而非单向“布道” 的关系, 弹幕族总体上是一个对外团结统一的利益体系。

\subsection{3. 观赏的共同体}

弹幕视频是时间轴的产物。它以时间为序列重新组织 了评论,而这种“时间”的概念又不停留在时/分这样抽象的 坐标上,而是将“时间”直接具象成为对应的画面,让用户的 评论针对画面产生聚合、共鸣与碰撞。自我认同的社交需 求在这种场景下被安抚。对弹幕族来说, 开着弹幕开视频 就像和朋友们一起边看边聊。弹幕满足了用户的归属感, 存在感和参与感。在相同情绪点, 适配弹幕的出现是发自 内心的惊喜感和相视一笑的契合感。人都有 $A$ 面和 $B$ 面, 一般社会人的 $\mathrm{A}$ 面身份后，是独处时的B面“人格”。孤独 的 $\mathrm{B}$ 面渴求文化内容消费, 渴求社交认同。比如, $\mathrm{B}$ 站弹

[7]丁依宁 (2015). 受众的表演与想象: 弹幕使用族群研究. 《新闻春 秋》.2015(04). 87-95.
幕中经常出现“一起刷剧的XX个小伙伴你们好”等打招呼 型弹幕。

《想象的共同体》一书中，作者本尼迪克特·安德森 认为, 民族本质上是一种想象的现代形式，它被想象为一 个本质上有限的政治意义上的共同体，而这一共同体的产 生离不开意识形态和现代语言团体所参与的社会创造(本 尼迪克特. 安德森,1983)。在网络时代这一观点依然适用, 信息技术的发展及互联网衍生服务为构建群体的想象(如 ACG 族群)提供了技术支持。具体到弹幕使用族群而言, 用户观看弹幕视频是为了寻找一种共同参与的围观感, 并 融入 $B$ 站这样的一个观赏共同体社区。

“蟾宫曲原野”在访谈表示: “大家一起看同一个视频, 一边抒发自己的意见, 一边看着别人的吐槽, 这种参与度 和互动感, 是你一个人静静的下载看视频不能比拟的。那 种知道“我不是一个人”的感觉很好。”

\subsection{4. 二次创作}

二次创作不同于剽窃, 是指使用了已存在著作物的文 字、图像、影片、音乐或其他艺术作品, 在其基础上进行 改编、仿作或发展而产生出新的作品。具体到弹幕使用族 群而言，他们的“二次创作”主要分为以下两种：

(1)弹幕的二次创作

在B站看视频, 经常在弹幕中发现“从电影院回来二刷 弹幕版”, “从优酷过来看弹幕”等评论。根据霍尔的编码 与解码理论, 编码的信息一旦进入到流通环节, 编码者就 失去了控制权，接收者在获得信息的同时根据自己的“库 存知识”进行解码, 再创造出新的信息意义。弹幕族就是 这样, 以原有的视频文本为基础, 根据个人主观情感色彩, 通过弹幕将评论叠加到视频上, 按照自身喜好对原有.视 频文本进行解码和再生产。视频内容经过弹幕加工后往往 会在原有的基础上发生一定程度的扩展、延伸和转移, 产 生新的文本意义。其中一个典型的表现就是“空耳”。“金 䇴”在访谈中表示:

金箔: B站的空耳很搞笑，我看《小林家的龙女仆》 的时候，片头OP（片头曲）里有一句“ちゅうちゅうYeah 亲亲Yeah Without you" (日式英文发音), 空耳之后就变成 了“舅舅爷喂了猪”，大家都在弹幕里刷这句话，类似这个 
动漫的黑话吧, 没看过的人进来可能一头雾水, 但是我们 这些一直追番的就会觉得很好笑。

(2)视频的二次创作

除却用添加弹幕的方式对视频内容文本进行颠覆性 解构, B站还聚集了一大批优秀的UP主, 对既有的视频素 材进行剪接重组, 赋予其截然不同的全新含义。

B 站最具有代表性的视频二次创作内容即 "鬼畜 " 视频。鬼畜视频指通过视频/音频剪辑, 用频率极高的重 复画面或声音组合成一段节奏配合音画同步的一类视频。 鬼畜视频通过UP主的“剪刀手”, 形成了完全不同于原素材 的含义, 以达到洗脑或喜感效果, 如红极一时的“极乐净 土”系列和长盛不衰的金切垃系列等。同样, 也有很多弹 幕族喜欢把自己喜爱的视频改编成鬼畜模式。

琳酱: 我在B站看《琅邪榜》周边的时候, 有个UP传 的视频叫《誉王什么的不干啦》, 就是把誉王的一些表演 鬼畜化, 然后配了一个特别燃的音乐, 结果出来特别搞笑, 我有一阵几乎每天都刷一遍, 笑的我肚子疼。还有之前的 雪姨系列那个 ‘你有本事开门啊', 也特别火, 看了以后都 不能直视原剧(《情深深雨蒙蒙》)了。UP主们太有才了。

对弹幕族而言, 鬼畜破坏了原有的符号系统, 与原型 形成巨大的反差感和对比度, 这种彻底解构结合着满屏弹 幕, 营造出独特环境下的颠覆功能。

\subsection{5. 弹幕视频内容的信息扩大化}

当视频中出现一些隐含的背景知识或者专业词汇时, 通常会有用户发弹幕进行解释说明。如B站常出现的一条 弹幕是“暂停看红字君科普”; 而当视频中将出现惊悚暴力 画面, 或者迎来剧情小高潮时, 会出现大批有秩序的弹幕 遮挡血腥画面或进行高能预警等。

谢坤: 有许多在 $\mathrm{B}$ 站看视频的人经常会 $\mathrm{N}$ 刷视频, 比 如我, 第一遍看的时候看视频内容, 二刷三刷的时候看弹 幕。因为视频展现的是创作者的想法, 弹幕传递的是观众 的意见。我在 $\mathrm{B}$ 站经常觉得同样的视频内容配上不同的弹 幕完全就像没看过一样, 能发掘出很多新的乐趣和信息。 至于观众用这些信息来干嘛, 是找共鸣, 找乐, 还是别的 什么, 因人而异, 每个人的点不一样, 需求的东西也不一 样。

\subsection{6. 在弹幕中寻找共同回忆}

中国的 $80 、 90$ 后是在电视、手机、互联网等电子产品 伴随下成长起来的, 他们的成长环境深受媒介影响, 与上 一代相比, 他们有着更多关于电子影像图景的共同回忆。 在访谈过程中, 访谈对象认为除了大同小异的校园生活, 他们的共同回忆中重要的组成部分还包括听过的歌, 看过 的动漫、影视剧, 甚至是某些令人记忆深刻的影视角色。 蟾宫曲原野: 我喜欢在 $\mathrm{B}$ 站看老剧, 能从里面发现了很多 以前忽略的乐趣。比如《铁齿铜牙纪晓岗》系列里, 大家 喜欢把纪晓岗和和珅拉 CP, 会在纪晓岗感情戏份刷“和宝 宝要吃醋了”, 还有各种恶搞, 看起来很欢乐。还有就是 小时候看不出来的一些桥段的漏洞和bug会被弹幕狂吐槽, (看视频的人) 年纪差不多, 一起看会感觉很温暖。

\section{4. 结论}

本研究以布尔迪厄场域理论为主要框架, 通过个案分 析、深度访谈加调查问卷辅助的研究方法, 从弹幕视频网 站、弹幕视频和弹幕使用族群三个维度展开分析, 分析中 国弹幕视频网站的文化场域是如何建构的。

布尔迪厄指出, 从场域的视角思考就是从关系的角度 思考。场域内各要素间的相互关系是建立场域的基础。具 体到本文而言, 弹幕视频网站提供了文化生产的场域空间, 弹幕视频语言系统的排他性将该场域和其他场域区隔开 来, 而弹幕族作为场域内的主要行动者, 团结又斗争地参 与者B站文化场域的建构。

$\mathrm{B}$ 站作为一个有限生产的文化场域, 在发展过程中体 现出区别于其他场域的自主性。B站丰富的内容资源、范 围广泛而条目细分的资源是其赖以生存的基础。其对自主 投稿视频的高质量要求和良好的互动反馈机制保障了用 户的观看体验, 也为 $\mathrm{B}$ 站视频内容注入其他视频网站难以 比肩的创造性和活力。其次, 从准入机制看, B站严格的 会员制度和完善的社区规则构成了该场域独特的进入壁 垒, 有利于更好地保证弹幕视频网站的区隔性和个性化, 凝聚一批高黏性用户。另一方面, B站还通过一系列丰富 的线上线下活动加强用户凝聚力, 不断扩大用户群。

弹幕视频排他性的话语体系构成了其独特的表达代 码。对场域外部用户来说, 弹幕语言是一种干扰性噪音, 而弹幕族则利用独特的弹幕语言来划分族群界限, 满足交 流需要和情感宣泄, 通过打破原有的语言规则以获得乐趣。 弹幕语言的核心风格是吐槽, 弹幕中言辞犀利的吐槽能让 用户获得一种错位幽默感, 好的吐槽甚至会激发用户彼此 间的语言创造力, 在弹幕中接力竞争。

$\mathrm{B}$ 站的弹幕族则呈现出年轻化、高学历、女性为主的 趋势。对弹幕族来说, 用户行为角色的模糊使得场域中生 产者和消费者间的“同源性”变得更加明显, 弹幕族在观看 弹幕视频中获得了“观赏共同体”的围观体验和群体内部 强烈的认同感。弹幕族通过弹幕生产和消费进行二次创作, 寻找集体记忆, 按照与 $\mathrm{B}$ 站相契合的惯习建构并维护 $\mathrm{B}$ 站 的场域生态, 在总体上形成了一个团结的利益体系。但同 时, 由于用户间存在次元壁、认知水平等各方面的差异, 弹幕族内部的联系又是松散的, 体现出场域的斗争性特征。

\section{参考文献}

[1] 本尼迪克特.安德森(2016). 《想象的共同体》.(吴睿人译). 上海:上海人民出版社.(原著出版于1983年)。

[2] 陈一, 曹圣琪, 王峡(2013).透视弹幕网站与弹幕族：一个 青年亚文化的视角.《青年探索》.2013(06).19-24。

[3] 陈席元(2014).弹幕话语建构的青年亚文化网络社群研究一 一以哔哩㗪哩对 keyki事件反应为例 J. 《电脑知识与技 术》.2014(20)67-69。

[4] 常宝(2011). 从布迪厄的“文化资本”理论谈族群文化的发展 问题.《西北民族研究》2011(03).32-36。 
[5] 陈联俊 (2012).网络社会“微博”與论场域的生成与引导.《社 会主义研究》.2012(06).62-66。

[6] 陈松松, 何天天(2014).弹幕视频一一小众网民的互动新形 式.《新闻世界》.2014(6).168-169。

[7] 丁依宁(2015).受众的表演与想象：弹幕使用族群研究.《新 闻春秋》，2015(04).87-95。

[8] 付晶晶(2016).新媒体时代的弹幕文化现象分析. 《南京邮电 大学学报》.2016(02).9-16。

[9] 高宣扬(2004). 《布迪厄的社会学》.上海: 同济大学出版社。

[10] 高雪(2015). 《抵抗与收编: 弹幕亚文化与主流文化的关 系研究》. 暨南大学硕士论文.广州。

[11] 江根源,季婧(2014).网络社区中的身份认同与网民社会结 构间的关联性.《新闻大学》.2014(02).83-92。

[12] 马志浩, 葛进平(2014). 日本动画的弹幕评论分析: 一种准 社会交往的视角.《国际新闻界》.2014(8).116-130。

[13] 李全生(2002). 布迪厄场域理论简析. 《烟台大学学 报》.15(2),2-27,146-150。

[14] 李琳琳(2015). 弹幕影像: 一场主体消解的狂欢机制. 《新 闻研究导刊》.2015(14).239-240。

[15] 李慧(2016).弹幕视频的传播现象研究.吉林大学硕士论文. 长春。

[16] 罗德尼·本森, 韩纲 (2003). 比较语境中的场域理论.《新 闻与传播研究》2003(01),2-23。

[17] 刘晗(2010). 福柯话语理论中的控制与反控制. 《兰州学 刊》.2010(04).204-208。

[18] 刘成富(2005).《科学的社会用途》.南京: 南京大学出版 社.17-19。

[19] 皮埃尔·布迪厄(2001). 《艺术的法则:文学场的生成与结构》 北京:中央编译出版社. (原著出版于1993年)。

[20] 让·鲍德里亚(2014). 《消费社会》(第四版).(刘成富,全志钢 译).南京: 南京大学出版社.(原著出版于1976年)。

[21] 宋雯(2009). 从霍夫斯塔德文化维度理论看汉英文化差异. 《安徽文学》.2009(06).75。

[22] 王雨辰(2001).略论葛兰西的市民社会与文化领导权理论. 《广西大学学报》2001(03),33-38。

[23] 韦文娟(2013). 简述霍尔高低语境文化在跨文化交际中的体 现.《现代企业教育》.2013(07).27-28。

[24] 王玉(2016).《从“自我认同”到“群体认同”-弹幕网站“迷群” 的“身份认同”》.安徽大学硕士论文.安徽。

[25] 谢娟,王晓再(2013). 场域理论视角下的微信“威胁论”解读. 《编辑之友》.2013(12).53-55。
[26] 谢梅, 何炬, 冯宇乐(2014).大众传播游戏理论视角下的弹 幕视频研究.《新闻界》.2014(2)37-40。

[27] 杨金凤(2010).《新媒介时代大众文化身份确认--以布迪厄场 域理论分析络文化的建构》. 山东师范大学硕士论文.济南市。

[28] 曾玉梅(2010). 《公民社会与网络社会两种理论路径下网络 社会交往的结构分析》.武汉大学博士论文.武汉。

[29] 张钗(2015). 弹幕视频的互动现状及发展策略. 《青年记 者》.2015（5）.65-66。

[30] 诸葛达维(2015). 互联网时代的弹幕电影分析一一基于互动 仪式链视角《新闻界》.2015(3).2-6。

[31］周舟(2015).《传播学视野下的网络青年亚文化一“弹幕文化” 解读》.西南大学硕士论文.重庆。

[32] Brown, Penelope and Stephen Levision(1987).Politeness: Some Universals in Language Usage. Cambrige: Cambrige University Press.

[33] Crystal, David(1998).Language Play. Chicago: The University of Chicago Press.

[34] Chi-hua Hsiao(2015).The verbal art of tucao and face-threatening acts in danmu screening. Chinese Language and Discourse,6(2).109-132.

[35] Goodwin, (1986).Audience Diversity, Participation and Interpretation. Special Issue of Text.6(3).283-316.

[36] Hamasaki M, Takeda H, Hope T, et al. Network analysis ofan emergent massively collaborative creation community, Proceedings of the Third International ICWSM Conference. 2009: 222-225.

[37] Lili Liu, Ayoung Suh, Christian Wagner(2016). Watching online videos interactively: the impact of media capabilities in Chinese Danmaku video sites. Chinese Journal of Communication(Taipei),7(06).283-303.

[38] Nakamura S, Shimizu M, Tanaka K(2008). Can social annotation support users in evaluating the trustworthiness of video clips? Proceedings of the 2nd ACM workshop on Information credibility on the web. ACM, 2(03). 59-62.

[39] Shen, Y., Chan,H., \&. Hung, I. Let the Comments Fly, The Effects of Flying Commentary Presentation on Consumer Judgment [C]. In Proceedings of the 35th International Conference on Information Systems (ICIS) conference, Auck-land.2014.

[40] Yuxiang Zhao, Jian Tang(2016).Exploring the Motivational Affordances of Danmaku Video Sharing Websites: Evidence from Gamification Design. Proceedings of HCI International Conference,11(02).1-13.

[41] Zechen Wu,Eisuke(2014).Correlation Analysis Between User's Emotional Comments and Popularity Measures, Proceedings of IEEEX plore. 